\title{
Hole Transport in Diketopyrrolopyrrole (DPP) Small Molecules: A Joint Theoretical and Experimental Study
}

\author{
$\mathrm{Zi} \mathrm{Li}^{\dagger}{ }^{\dagger} \mathrm{Xu} \mathrm{Z}_{\mathrm{Z}}{ }^{\dagger}{ }^{\dagger}$ Yuan Zhang, ${ }^{\ddagger}$ Cristiano F. Woellner, ${ }^{\ddagger}$ Martijn Kuik, ${ }^{\ddagger}$ Jianhua Liu, \\ Thuc-Quyen Nguyen, ${ }^{\ddagger}$ and Gang Lu* ${ }^{* \dagger}$ \\ ${ }^{\dagger}$ Department of Physics and Astronomy, California State University, Northridge, California 91330, United States \\ ${ }^{*}$ Department of Chemistry and Biochemistry, University of California, Santa Barbara, California 93106, United States
}

ABSTRACT: Charge transport in 3,6-bis[5-(benzofuran-2-yl)thiophen-2-yl]-2,5-bis(2-ethylhexyl)pyrrolo[3,4-c]pyrrole-1,4-dione $\left[\mathrm{DPP}(\mathrm{TBFu})_{2}\right]$ small molecules, a model donor material for organic photovoltaics, is studied by combined first-principles simulations and experiments. The dependence of the hole mobility on electric field, temperature, and molecular packing is examined in detail. Phononassisted hole transition rates and energetic disorder are identified as key intrinsic factors that control the hole mobility in DPP small molecules, with the latter playing a more important role than the former. In order to explain the substantial mobility differences between the as-cast and annealed films, we examine the effect of energetic traps on charge transport. For a trapping energy of $0.4 \mathrm{eV}$ and a trap density of $1.5 \times 10^{17} / \mathrm{cm}^{3}$, we find that $98 \%$ of the carriers

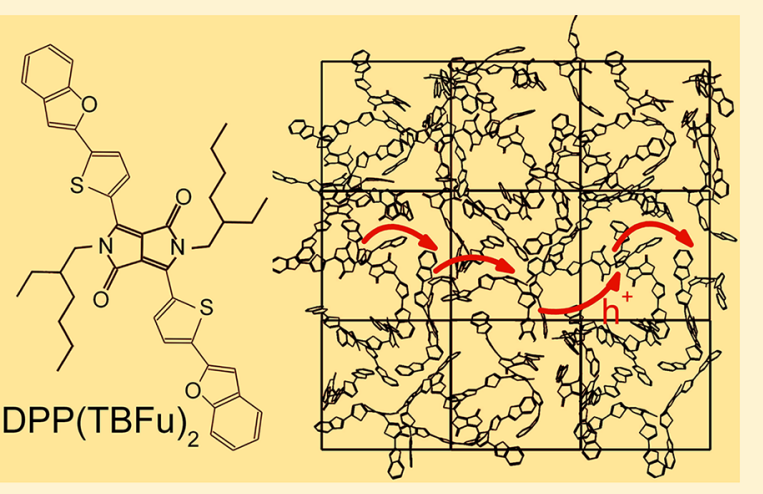
are trapped and the mobility drops by 2 orders of magnitude. With trap densities of $4.5 \times 10^{18} / \mathrm{cm}^{3}$ and $1.5 \times 10^{17} / \mathrm{cm}^{3}$ and trapping energy of $0.4 \mathrm{eV}$, we can reproduce the experimental mobilities for the as-cast and annealed films, respectively. Finally, we speculate on possible molecular defects that could act as traps for the holes.

\section{INTRODUCTION}

Solution-processable small molecules have attracted considerable interest in recent years as promising donor materials in organic photovoltaics (OPV). ${ }^{1-4}$ They offer some important advantages over conjugated polymers, such as facile synthesis and purification, greater tendency of self-assembly into ordered domains, and lack of undesirable batch-to-batch variations and end-group contaminations, which are detrimental to the performance of polymer-based OPVs. To be viable for largescale applications, however, the power conversion efficiency (PCE) of small-molecule-based solar cells should be substantially increased. Until now the morphological behavior in the blend and unbalanced charge transport of the donor and acceptor materials have been the major concerns in smallmolecule OPVs. ${ }^{5-7}$

In this paper, we report on a joint theoretical and experimental study of hole transport in disordered small molecules 3,6-bis[5-(benzofuran-2-yl)thiophen-2-yl]-2,5-bis(2ethylhexyl)pyrrolo[3,4-c]pyrrole-1,4-dione $\left[\mathrm{DPP}(\mathrm{TBFu})_{2}\right]$, whose molecular structure is shown in Figure 1a. A recent advance in small-molecule OPV has been reported in which $\mathrm{DPP}(\mathrm{TBFu})_{2}$ had been used as a donor material in bulk heterojunction $(\mathrm{BHJ}) \mathrm{OPV}$, resulting in a PCE of as much as $4.4 \%$. ${ }^{8,9}$ Our focus here on disordered small molecules is motivated by the fact that most OPVs consist of mixed ordered and disordered domains. Even in highly ordered films, amorphous or otherwise disordered domains nonetheless exist, most prominently in the form of amorphous pockets and/or grain boundaries. ${ }^{10}$ As a result, two charge-transport mechanisms coexist in OPVs. In the ordered domains, a bandlike transport mechanism is believed to operate, leading to higher carrier mobilities. ${ }^{11-13}$ In the disordered domains, on the other hand, charge transport proceeds as an incoherent random walk, weighted by phonon-assisted transition rates. ${ }^{14,15}$ Although the relative importance of the two mechanisms remains to be established, it has been argued that the overall carrier mobilities are controlled by the mobilities of the disordered domains. ${ }^{10}$ Furthermore, it has been demonstrated that owing to the strong electron-phonon coupling, the bandlike description of organic crystals could break down and the wave functions of charge carriers would become localized, originating from the dynamic disorder in the intermolecular coupling. ${ }^{11,16}$ Hence, charge transport in organic crystals would be similar to that in disordered materials. Consequently, it is of scientific and technological importance to understand charge transport in disordered organic materials, to elucidate factors controlling carrier mobility, and, it is hoped, to predict carrier mobility from first principles: a critical step toward computational design of OPV materials.

There are two types of disorder-static and dynamic disorder-influencing charge transport in organic semiconductors. The former refers to structural randomness and the latter

Received: October 22, 2012

Revised: March 11, 2013

Published: March 12, 2013 
originates from strong electron-phonon coupling. The division between static and dynamic disorder is somewhat porous, but in general the former is primarily associated with atomic structure and the latter with temperature. To describe the static disorder, phenomenological theories ${ }^{17-23}$ have been proposed to determine carrier hopping rates between molecular sites based on Miller-Abrahams ${ }^{24}$ and Marcus ${ }^{25}$ formalisms. Notably, recent work has suggested that deviations from these hopping rates can be observed from first-principles simulations. ${ }^{26}$ To capture the dynamic disorder, model Hamiltonians such as Holstein and Peierls types have been widely used but with mixed success, ${ }^{27-29}$ partly because these model Hamiltonians are based on perturbation theories and thus are restricted to specific ranges of microscopic parameters. More importantly, the aforementioned models are not materialspecific and contain empirical parameters that need to be predetermined; thus they lack predictability that is crucial for computational materials design. To overcome this key problem, we have recently developed a first-principles method that can predict carrier mobility in disordered semiconductors without empirical input or adjustable parameters. ${ }^{30}$ This method treats both dynamic and static disorder at the same footing and does not involve perturbation theories or harmonic approximation for phonons. In conjunction with a master equation approach, this method allows us to study charge transport in length scales that are relevant to realistic devices.

This paper is organized as follows. First, the computational method for a first-principles simulation of the mobility is briefly discussed; more details can be found in the Appendix and in ref 30. Subsequently, the drift-diffusion model for current densityvoltage $(J-V)$ behavior is introduced, after which the outcome of the first-principles simulation and the drift-diffusion modeling is analyzed. The simulation results are then compared with experimental data of as-cast and annealed hole-only diodes. It is found that, in order to describe the experimental data, trap levels need to be considered in the simulations. Finally, speculations are made on the origin of the traps.

\section{COMPUTATION METHOD AND PARAMETERS}

To model charge transport in disordered small molecules, we use a first-principles-based multiscale method ${ }^{30,31}$ that combines Born-Oppenheimer molecular dynamics (BOMD) for determining ground-state wave functions and energies, nonadiabatic molecular dynamics (NAMD) ${ }^{32}$ for evaluating the phonon-assisted transition rates between the charge carrier states, and the master equation simulation to calculate the carrier mobilities. The system of interest $(190 \mathrm{~nm}$ in each dimension) is divided into $100 \times 100 \times 100$ cubes for which the carrier mobilities are determined as a function of temperature, electric field, and molecular packing. The BOMD is performed in one of the $10^{6}$ cubes, referred to as the home cube, whose electronic wave functions and energies are used to generate the wave functions and energetic disorder in the other cubes. Specifically, the wave functions in each cube are selected from those in the home cube at a random BOMD snapshot, and they are then randomly rotated before being assigned to each cube. This is an approximate scheme to produce a random wave function network that resembles the true wave functions of the entire disordered system (which are impossible to calculate otherwise). The justification and validation of this scheme can be found elsewhere. ${ }^{30}$ The NAMD is subsequently carried out for the home cube to determine the transition rates between these wave functions.
The NAMD captures the phonon-assisted hole transitions between localized electronic states in the disordered molecules. Finally, based on these transition rates, the master equations are solved for the entire system to determine the carrier mobilities. In the Appendix, we provide a brief description of the multiscale method and the relevant computational parameters. The method has been applied to study carrier mobility in disordered semiconducting polymers ${ }^{30}$ and conjugated polyelectrolytes (CPEs). ${ }^{33}$

In crystalline organic semiconductors there are two types of molecular packing: slipped-cofacial and herringbone. ${ }^{14,34-36}$ In slipped-cofacial packing, the backbones of the molecules are parallel to each other, while in the herringbone packing, the neighboring backbones are tilted with each other for some angles. Since the precise molecular packing is not well characterized in disordered $\mathrm{DPP}(\mathrm{TBFu})_{2}$ films, it is reasonable to assume that the molecular packing may contain both herringbone and slipped-cofacial motifs, accompanied by random translations and rotations of the backbones. To model such structures, we construct the home cube with six $\mathrm{DPP}(\mathrm{TBFu})_{2}$ molecules (612 atoms) arranged differently to generate two representative structures as shown in Figure 1b,c. In structure I, we align the molecular backbones along the same
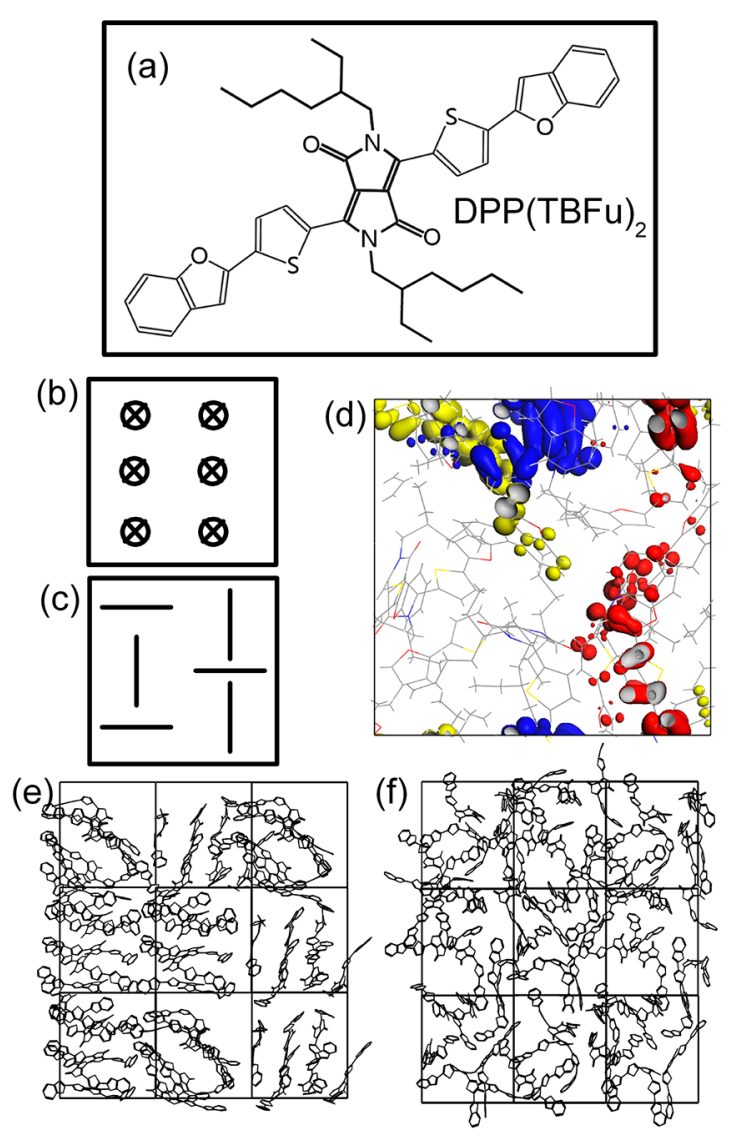

Figure 1. (a) Atomic structure of $\mathrm{DPP}(\mathrm{TBFu})_{2}$ molecule. (b, c) Molecular packing of structures I and II, respectively; $\otimes$ represents the backbone of the $\mathrm{DPP}(\mathrm{TBFu})_{2}$ molecule. (d) Structure II in the home cube superimposed with the wave functions of three highest occupied states; the isosurfaces (blue, yellow, and red) correspond to a value of $0.01 \mathrm{e} / \AA^{3}$. (e, f) Ttypical atomic structures in $3 \times 3 \times 1$ cubes generated from the $\mathrm{MD}$ simulations for structures I and II, respectively; only the backbones are shown. In each of the nine cubes, structure II is more disordered than structure I. 
direction to resemble the slipped-cofacial packing, while in structure II, the neighboring backbones are orientated perpendicularly to each other, similar to the herringbone packing. It is hoped that simulations of these two representatives could capture the essence of charge transport in the disordered molecules. Subject to subsequent molecular dynamics (MD) simulations, the molecules in the home cube will translate and rotate relative to each other. In Figure 1d, we display the wave functions of three highest occupied states, which are localized in the home cube; the positions of the carriers can thus be estimated from the localized wave functions. To construct the macroscopic system, we randomly select snapshots of atomic structure from the MD trajectory and rotate them randomly before placing them into different cubes. This is how disordered cofacial slip-stacking and herringbone packing structures shown in Figure 1e,f were generated.

A typical current density-voltage $(J-V)$ characteristic of the organic diode consists of drift current and diffusion current contributions. In general, at voltages below the built-in voltage $\left(V_{\mathrm{bi}}\right)$, the diffusion current dominates with a proportionality to the carrier density gradient. At voltages higher than $V_{\mathrm{bi}}$, the drift current takes over and the $J-V$ curve is described by a spacecharge-limited current (SCLC), which originates from the fact that the accumulated space charge inhibits further carrier injection until an equilibrium is reached inside the material with a uniform current density and a nonuniform electric field distribution. ${ }^{37}$ Therefore, in order to model $J-V$ curves for the entire voltage range, the well-established drift-diffusion model ${ }^{38}$ is used here by considering both current contributions: ${ }^{20,38-40}$

$$
\begin{aligned}
& J=p(x) \mathrm{e} \mu[E(x)] E(x)-\mathrm{e} D(x) \frac{\mathrm{d} p(x)}{\mathrm{d} x} \\
& \frac{\mathrm{d} E}{\mathrm{~d} x}=\frac{\mathrm{e}}{\varepsilon_{0} \varepsilon_{\mathrm{r}}} p(x) \\
& V=\int_{0}^{L} E(x) \mathrm{d} x
\end{aligned}
$$

where $x$ is the distance from the injecting electrode and $p(x)$ and $E(x)$ are the spatial-dependent carrier density and electric field. The diffusion coefficient $D(x)$ is assumed to follow the classical Einstein relationship, $D=k_{\mathrm{B}} T \mu / \mathrm{e}$, as suggested in Wetzelaer et al. ${ }^{41} \varepsilon_{0}$ is the vacuum permeability, $\varepsilon_{\mathrm{r}}$ is the relative dielectric constant of the molecule (a typical value for organic materials is 3 ), and $L$ is the thickness of the semiconductor film sandwiched between electrodes. The first and second terms in eq 1 correspond to the drift and diffusion current, respectively. In principle, the mobility $\mu$ should also be a function of carrier density. ${ }^{42}$ However, the mobility has been found to be independent of carrier density in the range $10^{14}$ $10^{16} \mathrm{~cm}^{-3}$ for polymers, ${ }^{42}$ which is in the similar range of carrier density for $\mathrm{DPP}(\mathrm{TBFu})_{2}$. Thus for simplicity, we assume the hole mobility of $\mathrm{DPP}(\mathrm{TBFu})_{2}$ to have an equally weak density dependence. Equations 1-3 are solved numerically following the method of van Mensfoort and Coehoorn. ${ }^{38}$

\section{CARRIER MOBILITIES FOR TRAP-FREE MATERIAL}

It is known that carrier mobility in disordered semiconductors is proportional to thermal equilibrium transition rates. ${ }^{30}$ According to eq 12, the thermal equilibrium transition rates depend on two quantities, the intrinsic transition rates $W_{i j^{\prime}}{ }^{0}$ and the energy difference between two relevant states where the transitions take place; the latter is also referred to as energetic disorder $(\sigma)$. The larger transition rates and smaller energetic disorder would lead to higher carrier mobilities. Note that the intrinsic transition rates also depend on the energy disorder indirectly because they are related to the carrier wave function coefficients whose evolution equation (eq 8) contains the energies $\varepsilon_{k}$. In Figure 2 and Table 1, we show the distribution
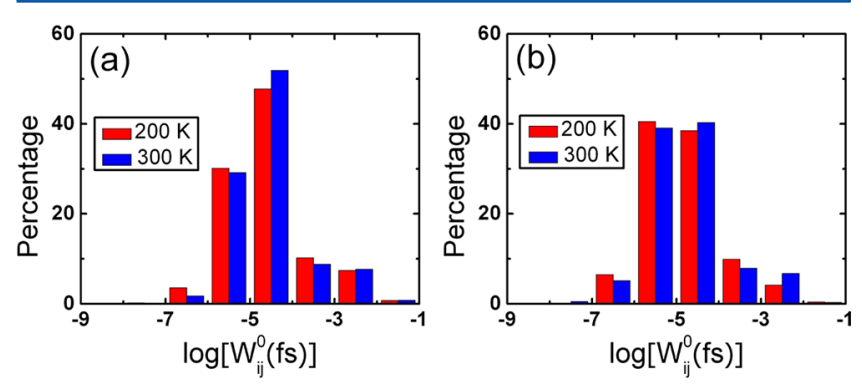

Figure 2. Percentage distribution of logarithm of intrinsic transition rates of (a) structure I and (b) structure II at $200 \mathrm{~K}$ (red) and $300 \mathrm{~K}$ (blue).

Table 1. Standard Deviation of HOMO Energy $\sigma$ of Structures I and II at Different Temperatures ${ }^{a}$

$\begin{array}{lcc} & \text { at } 200 \mathrm{~K} & \text { at } 300 \mathrm{~K} \\ \text { structure I }(\sigma) & 0.052 & 0.062 \\ \text { structure II }(\sigma) & 0.073 & 0.077 \\ & & \\ \text { structure I }\left(\sigma / k_{\mathrm{B}} T\right) & 3.0 & 2.4 \\ \text { structure II }\left(\sigma / k_{\mathrm{B}} T\right) & 4.2 & 3.0\end{array}$

${ }^{a}$ Both $\sigma$ (in electronvolts) and $\sigma / k_{\mathrm{B}} T$ are listed.

of the intrinsic transition rates as well as the energetic disorder as a function of temperature and molecular packing. Here the energetic disorder is represented by the standard deviation of the highest occupied molecular orbital (HOMO) energy $\sigma$ at different BOMD snapshots; we focus on the HOMO level because it has the greatest contribution to hole mobility among all valence states, dictated by the Fermi-Dirac statistics. Both the static and dynamic disorder can influence $W_{i j^{\prime}}{ }^{0}$ and $\sigma$, thus the mobility. The carrier mobility also depends on physical factors such as electric field, temperature, molecular packing, and traps for carriers, to name a few. ${ }^{14}$ In this section, we examine the mobility dependence on these factors in an effort to establish the connections between them and $W_{i j^{\prime}}{ }^{0}$ and $\sigma$.

Electric Field Dependence. As shown in Figure 3, under a uniform electric field, the mobility follows approximately the well-known Poole-Frenkel form, $\mu \propto \exp [\gamma(T) \sqrt{ } E]$ over an extended range of electric fields. ${ }^{43-45}$ The Poole-Frenkel relationship means that $\log \mu$ is proportional to $\sqrt{ } E$ and the proportional constant is $\gamma(T)$. However, we also observe notable deviations from the Poole-Frenkel form, especially at low fields. More specifically, the calculated $\log \mu$ curves deviate from the straight lines with reduced slopes at lower fields. It turns out that the deviations have an important consequence on comparisons between calculated and experimental zero-field mobilities, as described below. Deviations from the PooleFrenkel form at low electric fields have also been observed in the Gaussian disordered model (GDM). ${ }^{17}$ At high electric fields, we find that the mobility becomes temperatureindependent and the curves in both panels of Figure 3 are converging at high electric fields. This observation is explained 

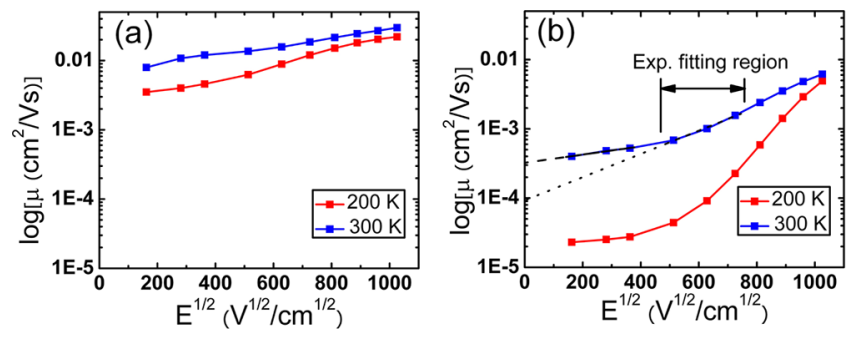

Figure 3. Logarithm of hole mobility of (a) structure I and (b) structure II versus the square root of applied uniform electric field at $200 \mathrm{~K}$ (red) and $300 \mathrm{~K}$ (blue). The dotted line in panel b indicates an extrapolation to zero electric field from the experimental fitting region of the fields; the dashed line represents an extrapolation to zero electric field from the first-principles results.

below. Calculated zero-field mobilities for the trap-free systems are summarized in Table 2.

Table 2. Zero-Field Mobilities for Trap-Free Structures I and II at Different Temperatures from First-Principles Simulations

\begin{tabular}{lcc} 
& \multicolumn{2}{c}{ zero-field mobility, $\mathrm{cm}^{2} /(\mathrm{V} \cdot \mathrm{s})$} \\
\cline { 2 - 3 } & at $200 \mathrm{~K}$ & at $300 \mathrm{~K}$ \\
structure I & $2.9 \times 10^{-3}$ & $5.2 \times 10^{-3}$ \\
structure II & $2.0 \times 10^{-5}$ & $3.1 \times 10^{-4}$ \\
\hline
\end{tabular}

Molecular Packing and Disorder. Molecular packing and degree of disorder are obviously related, although their connection is not always quantifiable. Here we characterize the molecular packing and disorder by two parameters: structural deformation and wave function localization length. When the molecule deforms, the atoms tend to deviate from the backbone plane; hence we can quantify the molecular deformation via

$$
\delta d=\left(\frac{1}{N} \sum_{i=1}^{N} d_{i}^{2}\right)^{1 / 2}
$$

where $d_{i}$ represents the atomic displacement from the backbone plane and $N$ is the number of atoms in the backbone of the molecule.

Wave function localization is a consequence of structural disorder and hence the localization length of wave functions could indicate the degree of disorder. Here we define the localization length of a wave function $i$ as $\delta \mathbf{r}_{i}$ :

$$
\delta \mathbf{r}_{i}^{2}=\int \rho_{i}(\mathbf{r})\left(\mathbf{r}-\overline{\mathbf{r}}_{i}\right)^{2} \mathrm{~d} \mathbf{r}
$$

where $\mathbf{r}$ is the electron coordinate, $\rho_{i}(\mathbf{r})=\left|\phi_{i}(\mathbf{r})\right|^{2}$ is the charge density of state $i$, and $\overline{\mathbf{r}}_{i}=\int \rho_{i}(\mathbf{r}) \mathbf{r}$ dr $\mathbf{r}$ is the average carrier position. The localization length $\delta \mathbf{r}_{i}$ represents the spatial extension of the carrier wave function, which is smaller for a more disordered structure. The average values of structural deformation and localization length during the BOMD simulation are 0.81 and $3.1 \AA$ for structure $I$ and 0.96 and $2.9 \AA$ for structure II, as shown in Figure 4. Hence structure II is more disordered than structure I from both the atomic and electronic structure point of view. In this paper, we use structures I and II as examples to demonstrate the effect of static disorder on charge transport. We find that the mobility of structure I is greater than that of structure II, and at $300 \mathrm{~K}$, this
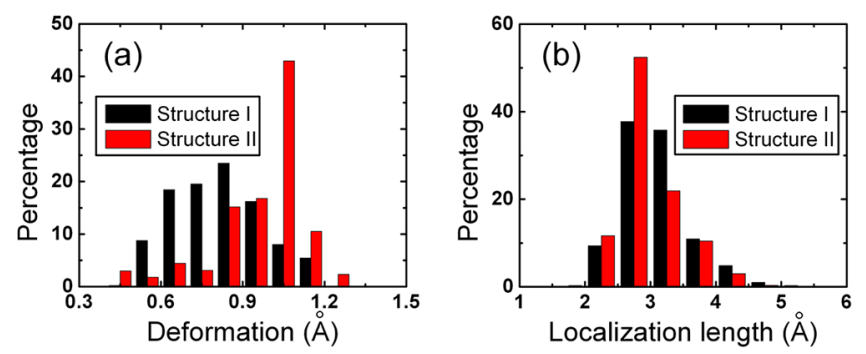

Figure 4. Percentage distribution of (a) structural deformation and (b) localization length for structure I (black) and structure II (red).

mobility difference could reach 1 order of magnitude. The results are consistent with the facts that the overall intrinsic transition rates of structure I are greater than those of structure II, and the energetic disorder $\sigma$ of structure II is higher than that of structure I. The experimental value of $\sigma$ at $300 \mathrm{~K}$ is 0.07 $\mathrm{eV}$, fitted from the Gaussian model, ${ }^{17}$ which is between the simulation results for structure I $(0.062 \mathrm{eV})$ and structure II $(0.077 \mathrm{eV})$.

To understand quantitatively the mobility dependence on molecular packings, we construct two models to disentangle the contributions from transition rates and energetic disorder. In the first model, we examine two fictitious materials IA and IIA; they have the same constant intrinsic transition rate but different energetic disorders corresponding to those of structures I and II at $300 \mathrm{~K}$, respectively. According to eq 15, the mobility should be proportional to the constant transition rate. It turns out that the mobility of material IA is 1 order of magnitude larger than that of IIA, entirely due to the energetic disorder (2.4 in IA and 3.0 in IIA), as shown in Figure 5a. In
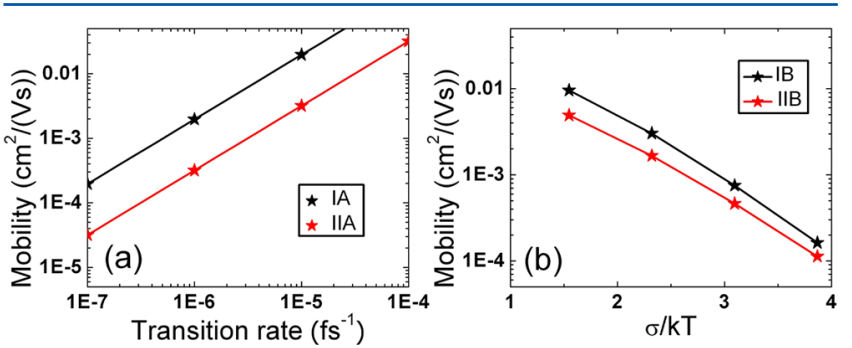

Figure 5. Mobilities of the fictitious materials IA and IB (black) and IIA and IIB (red): (a) from model calculations with a constant transition rate but different energetic disorders or (b) with the identical Gaussian distribution of HOMO energy but different transition rates. The lines serve as a guide to the eye. All mobilities are calculated with $\sqrt{ } E=360 \mathrm{~V}^{1 / 2} / \mathrm{cm}^{1 / 2}$.

the second model, we consider two other fictitious materials IB and IIB; they have an identical Gaussian distribution of the HOMO levels with a width of $\sigma$ (the standard deviation) but different intrinsic transition rates. As shown in Figure $5 b$, we find that the mobility $\mu$ increases with $\sigma / k_{\mathrm{B}} T$ and the mobility difference between the two materials is within a factor of 2 , entirely due to the transition rate difference. Therefore, on the basis of results from the fictitious systems, we conclude that an overall $\sim 20$ times difference in the mobility can be expected between structures I and II at $300 \mathrm{~K}$, consistent with the firstprinciples results shown in Table 2 . In addition, by inspecting Figure $5 \mathrm{~b}$, we find that the mobility can change by 2 orders of magnitude with $\sigma / k_{\mathrm{B}} T$ increasing from 1.5 to 3.8 , corresponding to an energetic disorder from 0.04 to $0.10 \mathrm{eV}$. 
Temperature Dependence. Dynamic disorder is primarily determined by temperature, which can affect both the electronic structure and energy levels of the molecules, and in turn change the intrinsic transition rates and energetic disorder. Hence, by examining temperature dependence of carrier mobilities, one can gain insights on how the dynamic disorder may influence charge transport in the disordered molecules. From both $\mu-E$ curves and zero-field mobilities, we find that the mobilities increase with temperature, consistent with the charge hopping model for disordered materials. To examine the effect of energetic disorder on mobility, it is more appropriate to focus on $\sigma / k_{\mathrm{B}} T$ because the thermal equilibrium transition rate depends on $\sigma / k_{\mathrm{B}} T$; the density of states (DOS) for the absorbed phonon is proportional to $\mathrm{e}^{-\sigma / k_{\mathrm{B}} T}$, thus a smaller $\sigma /$ $k_{\mathrm{B}} T$ leads to a larger phonon DOS and hence a higher probability for the carrier to absorb the phonon in order to overcome the energy barrier $\sigma$. Since $\sigma / k_{\mathrm{B}} T$ at $200 \mathrm{~K}$ is higher than that at $300 \mathrm{~K}$, the mobility at $200 \mathrm{~K}$ is lower. We have constructed similar models as above to examine temperature dependence of mobility, and we find that energetic disorder plays a much more dominant role than transition rates, as expected from eq 12 .

As displayed in Table 2, the zero-field mobility increases $100 \%$ and $1000 \%$ for structures I and II, respectively, with increasing temperature from 200 to $300 \mathrm{~K}$. The much greater mobility increase in structure II can be understood from the Arrhenius law $\mu \sim \exp \left(-\Delta / k_{\mathrm{B}} T\right)$, in which $\log \mu$ is proportional to $1 / T$ with the slope being the activation energy $\Delta$. Since greater static disorder in structure II leads to larger activation energy, ${ }^{15}$ its mobility increases much faster (see Figure $6 \mathrm{c}$ for the Arrhenius law). The thermal effect on mobility tapers off at high electric fields because the hopping energy barriers are effectively lowered by work done by the electric fields, and thus the thermal activation becomes less dominant.

Comparison of Mobilities to Other Organic Materials. The same theoretical method has been applied to poly(3hexylthiophene) (P3HT) polymer and conjugated polyelectrolytes previously. ${ }^{30,33}$ We find that the intrinsic transition rates are similar $\left(\sim 10^{-5} \mathrm{fs}^{-1}\right)$ among these organic semiconductors because of their similar $\pi-\pi$ stacking. However, the energetic disorder in these materials is different and plays a crucial role. $\mathrm{P} 3 \mathrm{HT}$ and the anionic $\mathrm{CPE}$ have a larger energetic disorder $(\sim 0.1 \mathrm{eV})$ than that of $\mathrm{DPP}(\mathrm{TBFu})_{2}$ and the cationic $\mathrm{CPE}(\sim 0.08 \mathrm{eV})$, and this difference is responsible for higher mobilities of the latter group. The greater energetic disorder in $\mathrm{P} 3 \mathrm{HT}$ is due to the fact that each thiophene ring can rotate relative to its neighbor rings, rendering a more flexible structure and thus a larger energy fluctuation. ${ }^{46,47}$ In contrast, in $\mathrm{DPP}(\mathrm{TBFu})_{2}$ and the cationic $\mathrm{CPE}$, the conjugation part (DPP core or fluorene) is less flexible, leading to a smaller energetic disorder and higher mobilities. In comparison with the conjugated polymers, the small molecules have identical structure units, hence a more uniform energy distribution. Therefore the energetic disorder in the small molecules is lower with generally higher mobilities. In the case of the anionic CPE, the dynamics of the counterions is responsible for the larger energetic disorder. Although these materials are very different from an experimental point of view, their charge-transport mechanism is similar and can be understood in terms of intrinsic transition rates and energetic disorder.

\section{EXPERIMENTAL METHODS}

In order to examine the hole transport in $\mathrm{DPP}(\mathrm{TBFu})_{2}$, single carrier diodes with the sandwich structure ITO/PEDOT-PSS/ $\mathrm{DPP}(\mathrm{TBFu})_{2} / \mathrm{Au}$ were fabricated in inert gas environment as follows: $50 \mathrm{~nm}$ of poly(3,4-ethylenedioxythiophene)-poly(styrenesulfonate) (PEDOT-PSS) hole injection layer was spin-coated atop patterned indium-tin oxide (ITO) substrates that were precleaned with sonication in soapy water, acetone, and 2-propanol sequentially. The PEDOT-PSS layer is intended to enhance the wettability and smoothness by offering a work function of $5.1 \mathrm{eV}$ to form an ohmic contact with the HOMO of DPP $(\mathrm{TBFu})_{2}(5.3 \mathrm{eV}$, as confirmed by ultraviolet photoemission spectroscopy $)^{8}$. The $\mathrm{DPP}(\mathrm{TBFu})_{2}$ active layers were attained by spin-coating from the chloroform solution filtered by a PTFE membrane filter with a concentration of 20 $\mathrm{mg} / \mathrm{mL}$, resulting in a film thickness of around $140 \mathrm{~nm}$. The final step of the device fabrication involved thermal evaporation of $80 \mathrm{~nm} \mathrm{Au}$ top cathode through shadow masks under pressure of $\sim 10^{-7}$ Torr. After the metal deposition was finished, the $\operatorname{DPP}(\mathrm{TBFu})_{2}$ devices were thermally annealed on a hot plate at $110{ }^{\circ} \mathrm{C}$ for $10 \mathrm{~min}$ and cooled down immediately before testing in nitrogen environment. The $J-V$ characteristics were recorded in a Lakeshore vacuum probe station by using a Keithley 4200 semiconductor parameter analyzer. Low-temperature measurement was performed by use of a customized cryogenic system purged with liquid nitrogen flux and controlled by a Lakeshore temperature controller.

\section{COMPARISON TO EXPERIMENT AND THE ROLE OF TRAPS}

Comparison between Theory and Experiment. The quantities of interest here are the zero-field mobility and $J-V$ curves. On the theory side, one first determines the zero-field mobility by first-principles calculations. On the basis of the calculated mobility, one then computes the $J-V$ curves using the drift-diffusion model as described earlier. Because the driftdiffusion model has limitations as applied to different materials and over an entire range of voltages, the theory has uncertainties in predicting $J-V$ curves. On the experimental side, on the other hand, one first measures the $J-V$ curves and then fits the curves to certain analytical models to extract the zero-field mobility. Thus in general, one would expect that the mobility is more reliable than the $J-V$ curves on the theory side, while the $J-V$ curves are more reliable than the mobility on the experimental side; one should keep this disparity in mind when comparing between theory and experiment.

Figure 6 displays the logarithmic $J-V$ characteristics of devices composed of (a) as-cast and (b) $110{ }^{\circ} \mathrm{C}$ annealed $\mathrm{DPP}(\mathrm{TBFu})_{2}$, measured at various temperatures. Temperature dependence of $J$ is observed, suggesting that charge transport is thermally activated and governed by disorder. The hole current displays a stronger voltage dependence at low temperature, as revealed by the curving-up deviation from the quadratic relationship at high voltages. The origin of the electric-field dependence is the presence of energetic disorder, which results in a localization of carrier states and hopping transport. ${ }^{48,49}$ Of importance is that the current of the annealed film increases by 1 order of magnitude when compared to the as-cast film and the difference is further enlarged at low temperature. By means of conducting atomic force microscopy, nanoscale film morphology of $\mathrm{DPP}(\mathrm{TBFu})_{2}$ is found drastically changed upon annealing, exhibiting an increase in crystallinity and 


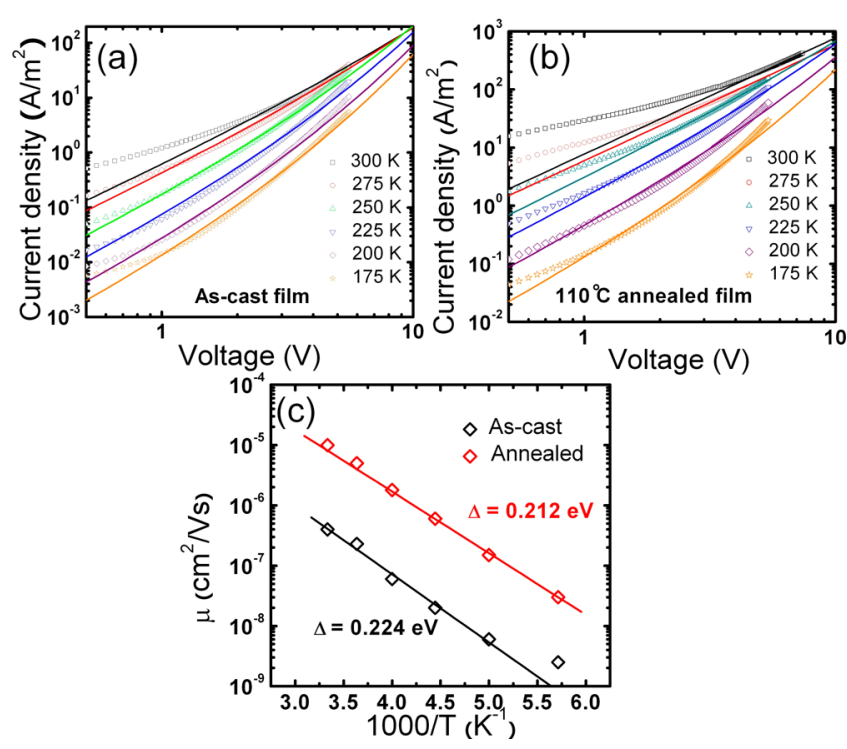

Figure 6. Experimental $J-V$ characteristics of (a) as-cast and (b) annealed films are shown with different symbols at different temperatures. Curves are the fits to the experimental data points following the Poole-Frenkel relationship. (c) Experimental zero-field mobilities as a function of temperature.

enlarged domain size. ${ }^{9,50}$ The enhanced hole transport associated with increased carrier mobility is thus likely attributable to the morphological improvement. The universality of this observation can be witnessed by many DPPcontaining small-molecule semiconductors. ${ }^{51}$

The fitting of the $J-V$ curves depends on the assumptions that both the Poole-Frenkel relationship and the MottGurney law $^{37}$ are valid in the voltage range where the experimental $J-V$ curves are fitted. With these assumptions, the following equations are used in the fitting, from which the key quantity $\mu_{0}$ can be extracted: ${ }^{44,52}$

$$
\begin{aligned}
& J=\frac{9}{8} \varepsilon_{0} \varepsilon_{\mathrm{r}} \mu \frac{V^{2}}{L^{3}} \\
& \mu=\mu_{0} \exp (\gamma \sqrt{E})
\end{aligned}
$$

where $E$ is the average electric field defined as $V / L$.

If the Poole-Frenkel relationship were valid over the entire voltage range after $V_{\mathrm{bi}}$, the fitting parameter $\mu_{0}$ would become the zero-field mobility. Otherwise, $\mu_{0}$ represents a finite field mobility. However, as indicated by our first-principles calculations and the GDM simulations by others, ${ }^{17}$ the Poole-Frenkel relationship is not valid at low voltages; thus one has to be cautious in interpreting $\mu_{0}$ as the zero-field mobility.

To extract the zero-field mobility of the annealed film at 300 $\mathrm{K}$, the fit is carried out over a voltage range of $3-8 \mathrm{~V}$ as shown in Figure $6 \mathrm{~b}$, corresponding to an electric field range of $\sqrt{ } E=$ $470-760(\mathrm{~V} / \mathrm{cm})^{1 / 2}$. However, as shown in Figure $3 \mathrm{~b}$, the log $\mu-\sqrt{ } E$ relationship over this range of voltages is different from that at lower voltages; the dotted line of the fit has a larger slope than the dashed line of the simulation. In other words, the fitted mobility is smaller than the simulated zero-field mobility. Specifically, the fitted mobility is $9.3 \times 10^{-5} \mathrm{~cm}^{2} /(\mathrm{V} \cdot \mathrm{s})$, substantially lower than the simulated mobility of $3.1 \times 10^{-4}$ $\mathrm{cm}^{2} /(\mathrm{V} \mathrm{s})$. Hence we believe that the zero-field mobility extracted from the experimental $J-V$ curve underestimates the real zero-field mobility, sometimes significantly. This partially explains the contrasting zero-field mobilities obtained by theory and experiment: experimental mobilities for the annealed film are $\mu_{300 \mathrm{~K}}=1.0 \times 10^{-5}$ and $\mu_{200 \mathrm{~K}}=1.5 \times 10^{-7} \mathrm{~cm}^{2} /(\mathrm{V} \cdot \mathrm{s})$, while mobilities for the as-cast film are $\mu_{300 \mathrm{~K}}=4.0 \times 10^{-7}$ and $\mu_{200 \mathrm{~K}}=$ $6.0 \times 10^{-9} \mathrm{~cm}^{2} /(\mathrm{V} \cdot \mathrm{s})$. The simulated mobilities of structure II are $\mu_{300 \mathrm{~K}}=3.1 \times 10^{-4}$ and $\mu_{200 \mathrm{~K}}=2.0 \times 10^{-5} \mathrm{~cm}^{2} /(\mathrm{V} \cdot \mathrm{s})$.

We have also used the $\mu(E)$ dependence from the Pasveer model $^{20}$ to fit the experimental $J-V$ curves; we find that with this $\mu(E)$ relationship, the fitted zero-field mobilities change only by $50 \%$. Owing to the existence of diffusion current at low voltages, the Pasveer model, which is based on the spacecharge-limited current, cannot reproduce the entire experimental $J-V$ curves either. Therefore, the uncertainties of fitting zero-field mobility persist in both Poole-Frenkel and Pasveer models. In addition to the underestimation due to fitting, discrepancies between simulation and experiment can also be attributed to the presence of traps in the experimental samples (particularly in the as-cast film), which is not considered in the present first-principles simulations. In the next section, we will show that the mobility discrepancies can indeed be accounted for by traps. Finally, in Figure 6c, the Arrhenius relationship $\mu$ $\sim \exp \left(-\Delta / k_{\mathrm{B}} T\right)$ is observed from the experimental mobilities with activation energy $\Delta$ of 0.21 and $0.22 \mathrm{eV}$ for the annealed and as-cast films, respectively.

Role of Traps in Charge Transport. Defects are common in organic materials, and their importance in OPV has been widely recognized. However, although defects have been studied for a long time, their chemical nature, and in particular to what extent certain defects could influence charge transport in disordered organic semiconductors, has not been well established. In fact, there is no direct experimental evidence that defects can actually trap carriers. In the following, we will examine how traps could affect carrier mobility without specifying the chemical nature of the traps; in other words, the traps may or may not be defects. We model the traps of carriers by elevating the HOMO energy levels in selected cubes, and we determine the corresponding carrier mobilities from the master equations. More specifically, we randomly select a number of cubes and uplift the HOMO level of each selected cube by a certain amount $\left(\Delta E_{t}\right)$. The energy increase $\Delta E_{\mathrm{t}}$ is termed as trapping energy in the following, and the traps are thus energetically favorable for the holes.

Since the chemical nature of traps is unknown, there is no consensus on the energy distribution of trap states. In other words, it is impossible to simulate the traps directly from first principles. Previously, various energy distributions of traps have been attempted, including Gaussian, exponential, and single discrete levels, for fitting experimental $J-V$ curves. $^{53-55}$ It has been shown that for a low carrier density material such as the one considered here, the Gaussian distribution of trap states yields a similar $J-V$ relationship as a single trap level. ${ }^{53,56}$ Therefore, in the following simulation we will assume the single trap level, which is the simplest model that can capture the essential physics and provide an order-of-magnitude estimate for the trap effect on the mobilities.

We calculate the carrier mobility for structure II at $300 \mathrm{~K}$, and the results are shown in Figure 7a. Here the trapping cube density $n_{\mathrm{t}}$ indicates the percentage of selected cubes from the entire system of $10^{6}$ cubes, and the trapping energy is defined as the energy increase relative to the global HOMO level. $n_{\mathrm{t}}$ is then divided by the volume of the home cube to give the trap density. We find that the traps could reduce carrier mobility by 

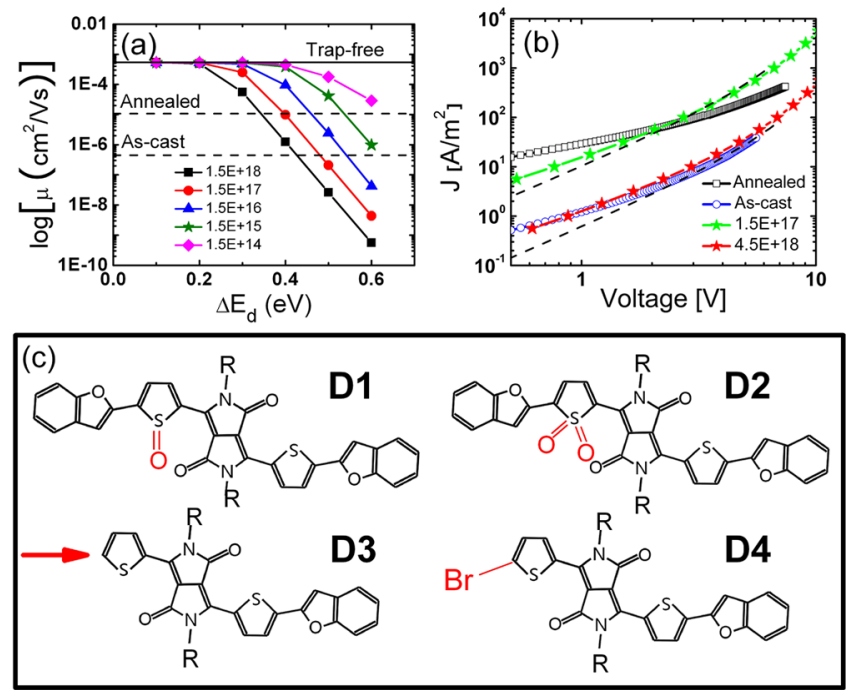

Figure 7. (a) Calculated mobilities as a function of trapping energy and trap density at $300 \mathrm{~K}$. Experimental mobilities at $300 \mathrm{~K}$ for annealed and as-cast films are shown by horizontal dashed lines. The simulated trap-free carrier mobility is indicated by the horizontal solid line. (b) Comparison between experimental (black and blue) and theoretical (red and green) $J-V$ curves. The theoretical results are for trap densities of $1.5 \times 10^{17} / \mathrm{cm}^{3}$ (green) and $4.5 \times 10^{18} / \mathrm{cm}^{3}$ (red) with a trapping energy of $0.4 \mathrm{eV}$. The theoretical $J-V$ curves without the diffusion current are shown as dashed lines. (c) Possible defect structures examined in the paper; red arrows and symbols highlight the defect sites.

many orders of magnitude depending on the trap density and trapping energy. For each trap density, there is a minimum trapping energy below which the traps have a negligible effect on mobility. This critical energy is defined as the onset energy, where the mobility curves start deviating from the trap-free mobility (solid horizontal line) in Figure 7a. The critical energy has to be much higher than the energetic disorder; otherwise the energy fluctuation would overrun $\Delta E_{\mathrm{t}}$ and detrap the carriers (for example, the HOMO level has an energy fluctuation as large as $0.25 \mathrm{eV}$ at $300 \mathrm{~K})$. We observe two general trends from Figure 7a: (1) For the same trapping energy, the larger the trap density, the lower the carrier mobility. (2) For the same trap density, the higher the trapping energy, the lower the carrier mobility; in particular, the mobility decreases exponentially with increasing trapping energy. These results are in a full agreement with the well-known single discrete trap level model. ${ }^{57}$

The intersections between mobility curves and the two horizontal dashed lines correspond to conditions under which the calculated mobility matches exactly the experimental mobility. We find that there are many possibilities (different combinations of trap density and trapping energy) that would yield exact agreement between the theoreticalal and experimental mobilities. This has important implications: (1) Without knowing the trap density and trap structure precisely in an experimental sample, it is impossible to reproduce the experimental mobilities from first principles. Of course, one can compute the trap-free carrier mobilities, as we have done here, to estimate an upper bound of the true mobilities. There are also instances where the trap-free mobilities are themselves useful. (2) The carrier mobilities depend sensitively on traps, and even in the well-annealed samples, traps could play an important role in charge transport.
By assuming trapping energies of 0.4 and $0.5 \mathrm{eV}$, we can estimate the corresponding trap densities at which the experimental mobilities match the simulations. The results are summarized in Table 3. We find that the trap densities in the

Table 3. Trap Densities at Which the Experimental Mobilities Are Reproduced by Simulations for Trapping Energy of 0.4 or $0.5 \mathrm{eV}$

\begin{tabular}{lcc}
\multicolumn{2}{c}{ trap density, $\mathrm{cm}^{-3}$} \\
\cline { 2 - 3 } & $0.4 \mathrm{eV}$ trapping energy & $0.5 \mathrm{eV}$ trapping energy \\
annealed, 300 K & $1.5 \times 10^{17}$ & $4.5 \times 10^{15}$ \\
as-cast, 300 K & $4.5 \times 10^{18}$ & $7.5 \times 10^{16}$ \\
annealed, 200 K & $1.5 \times 10^{16}$ & $3.0 \times 10^{14}$ \\
as-cast, 200 K & $4.5 \times 10^{17}$ & $3.0 \times 10^{15}$ \\
\hline
\end{tabular}

annealed film are 1 order of magnitude lower than those in the as-cast films for both temperatures and trapping energies. In addition, the hole trap densities at $300 \mathrm{~K}$ are 1 order of magnitude greater than those at $200 \mathrm{~K}$, which have a similar trend as observed in $\mathrm{OC}_{1} \mathrm{C}_{10}-\mathrm{PPV}$, where the effective electron trap densities are higher at higher temperatures. ${ }^{54}$ Our estimates in Table 3 are in line with the results reported in ref 58 that the trap density is $3 \times 10^{17} / \mathrm{cm}^{3}$ and the trapping energy ranges from 0.2 to $0.8 \mathrm{eV}$. In Figure $7 \mathrm{~b}$, we display the $J-V$ curves calculated with the drift-diffusion model and compare them with the experimental ones. The calculation is performed with trapping energy of $0.4 \mathrm{eV}$ and trap densities of $1.5 \times 10^{17} / \mathrm{cm}^{3}\left(n_{t}=0.1 \%\right)$ and $4.5 \times 10^{18} / \mathrm{cm}^{3}\left(n_{t}=3 \%\right)$ for annealed and as-cast samples, respectively. We find that the simulated $J-V$ curve matches very well to the experimental one for the as-cast film. For the annealed film, however, the calculated $J-V$ curve does not match as well to the experimental one, and we suspect that the leakage current in the annealed film may be the culprit for the discrepancy. The $J-V$ curves calculated without the diffusion term in eq 1 are also shown in Figure $7 \mathrm{~b}$ as dashed lines; it is evident that the diffusion current contribution is important at low voltages.

To understand the trapping of the carriers, we calculate the carrier density distribution, that is, the partial concentration of $p_{n}$, in eq 14 . We find that with a trap density of $1.5 \times 10^{17} / \mathrm{cm}^{3}$ and a trapping energy of $0.4 \mathrm{eV}, 98 \%$ of all carriers are trapped. Because of the large trapping energy, these trapped carriers are practically immobile. For the same trap density but a trapping energy of $0.2 \mathrm{eV}$, only $2 \%$ of carriers are trapped, and thus the mobility drop is negligible. Since the traps are randomly selected, the trapped carriers are not uniformly distributed. In fact, the number of trapped carriers in each trapping cube depends on the HOMO levels of the neighboring cubes, as the detrapping probability scales exponentially with the HOMO energy differences between the trapping cube and its neighbors.

Finally, we speculate on possible defect structures that could give rise to deep trap states. We have considered four possible defects whose structures are shown in Figure 7c. Defects D1 and D2 are derived from oxidation of the sulfur atom in the thiophene ring with one and two $\mathrm{S}=\mathrm{O}$ bonds that have been observed experimentally in the thiophene molecules. Defect D3 corresponds to a missing benzofuran unit of the $\mathrm{DPP}(\mathrm{TBFu})_{2}$ molecule, and D4 corresponds to a Br-substituted D3 structure; both of them could be formed during synthesis. However, we find that all these defects have a lower HOMO level than that of the perfect $\mathrm{DPP}(\mathrm{TBFu})_{2}$ molecule. Therefore, they are not energetically favorable for holes, and they mainly act as 
scattering centers as opposed to traps for the carriers. Our findings are consistent with a recent review on this subject, which asserts that chemical contaminants from synthesis, vacancies, and molecular products from oxidation are not expected to be effective charge traps. ${ }^{11}$ Thus we believe there should be other molecular structures that serve as traps, but identification of them is beyond the scope of the present work.

\section{SUMMARY}

We have studied charge transport in $\mathrm{DPP}(\mathrm{TBFu})_{2}$ small molecules by combining experiments and first-principles simulations. The dependence of hole mobilities on electric field, temperature, and molecular packing is examined in detail. The mobility-electric field dependence shows an approximate Poole-Frenkel relationship, and the mobilities at low electric fields depend strongly on temperature and could vary by 1 order of magnitude between 200 and $300 \mathrm{~K}$. Molecular packing can change energetic disorder and thus carrier mobilities by more than 1 order of magnitude. Transition rates and energetic disorder are identified as key intrinsic factors controlling carrier mobilities in trap-free materials, while energetic disorder plays a far more important role than transition rates. We point out that the experimental fitting procedure could potentially underestimate zero-field mobility. We have simulated the effect of traps on charge transport by examining the mobility dependence on trap density and trapping energy. We find that there is a minimal trapping energy below which the traps have a negligible effect on charge transport. With a trapping energy of $0.4 \mathrm{eV}$ and a trap density of $1.5 \times 10^{17} / \mathrm{cm}^{3}, 98 \%$ of the carriers are trapped and the mobility drops by 2 orders of magnitude. With trap densities of $4.5 \times 10^{18}$ and $1.5 \times 10^{17} / \mathrm{cm}^{3}$ for as-cast and annealed samples, respectively, we can reproduce the experimental mobilities. Furthermore, we can reproduce the experimental $J-V$ curve for the as-cast film using the same trap density and trapping energy. Finally, we speculate on possible defects that may act as deep traps for the carriers.

\section{APPENDIX}

\section{Ab Initio BOMD Simulation}

The ab initio BOMD simulation is carried out for the home cube with the standard plane-wave pseudopotential approach and the generalized gradient approximation ${ }^{59}$ as implemented in the VASP code. ${ }^{6,61}$ The calculation is performed at the Gamma point only, and the energy cutoff of the plane-wave basis is $400 \mathrm{eV}$.

The lattice constant of the home cube is $19.0 \AA$, corresponding to a mass density of $1.1 \mathrm{~g} / \mathrm{cm}^{3}$, slightly smaller than the experimentally estimated density of $1.3 \mathrm{~g} / \mathrm{cm}^{3}$ in single crystalline phase. In general, the dimensions of the home cube should be chosen as large as computationally feasible, but the minimal dimensions should be larger than the intersite distance defined in the well-known Gaussian disordered model, ${ }^{17}$ which is about $16 \AA^{20}$ Isothermal-isobaric (NPT) classic MD is performed at $500 \mathrm{~K}$ to obtain disordered initial configurations for both packings. The initial configurations are then fully relaxed by static ab initio calculations before the ab initio BOMD simulations are carried out to bring the system to the desired temperature with repeated velocity scalings. The home cube is kept at $300 \mathrm{~K}$ for $500 \mathrm{fs}$ to reach thermal equilibrium. Finally, a microcanonical BOMD production run is carried out for $1000 \mathrm{fs}$. We have examined the autocorrelation function for $\mathrm{HOMO}$ energy during the micro-canonical MD run and found that the system is well equilibrated in $1000 \mathrm{fs}$. In the BOMD simulations, the time step is $1 \mathrm{fs}$.

\section{NAMD for Transition Rates}

On the basis of Kohn-Sham (KS) wave functions and energies determined at each BOMD time step, we can calculate the phonon-assisted electron transition rates between these KS states in the home cube by the NAMD approach; ${ }^{30}$ they are called intrinsic transition rates in the following. We expand the time-dependent one-electron wave function of the carrier, $\psi^{(\mathrm{i})}(\mathbf{r}, t)$ in terms of a full set of adiabatic KS orbitals $\phi_{j}[\mathbf{r}, \mathbf{R}(t)]$ at time $t$ :

$$
\psi^{(\mathrm{i})}(\mathbf{r}, t)=\sum_{j} c_{j}^{(\mathrm{i})}(t) \phi_{j}[\mathbf{r}, \mathbf{R}(t)]
$$

Here $\phi_{j}[\mathbf{r}, \mathbf{R}(t)]$ is the $j$ eigenstate of the KS Hamiltonian for the ionic configuration $\mathbf{R}(t)$, and $c_{j}^{(\mathrm{i})}(t)$ is the corresponding expansion coefficient. The superscript $\mathrm{i}$ signifies that at $t=0$, $\psi^{(\mathrm{i})}(\mathbf{r}, 0)=\phi_{i}[\mathbf{r}, \mathbf{R}(0)]$ is an initial condition. For simplicity, we assume that at $t=0$, the carrier is in a pure state $\phi_{i}[\mathbf{r}, \mathbf{R}(0)]$, and the subsequent motion of the ions evolves the state to a mixed state as in eq 7. The expansion coefficient thus represents the transition probability amplitude from the initial state $i$ to the state $j$. These transitions are assisted by the ionic vibrations, and thus the transitions are called phonon-assisted transitions. Substituting eq 7 into the time-dependent KS equation, one can obtain the following equation for the evolution of the coefficient $c_{j}^{(\mathrm{i})}(t):^{32}$

$$
\frac{\partial}{\partial t} c_{j}^{(\mathrm{i})}(t)=-\sum_{k} c_{k}^{(\mathrm{i})}(t)\left(\frac{i}{\hbar} \varepsilon_{k} \delta_{j k}+d_{j k}\right)
$$

where $\varepsilon_{k}$ is the energy of the $k$ th KS orbital, and $d_{j k}$ is the nonadiabatic coupling (NAC) between the $j$ th and $k$ th $\mathrm{KS}$ orbitals:

$$
d_{j k} \equiv\left\langle\phi_{j}\left|\nabla_{\mathbf{R}}\right| \phi_{k}\right\rangle \cdot \frac{\mathrm{d} \mathbf{R}}{\mathrm{d} t}=\left\langle\phi_{j}\left|\frac{\partial}{\partial t}\right| \phi_{k}\right\rangle
$$

Equation 8 is propagated over a 100 -fs-long trajectory with an initial condition $c_{i}^{(\mathrm{i})}(t=0)=\delta_{i j}$ imposed at each BOMD time step; the eigenvalues and NAC at each BOMD time step are used in the propagation of eq 8 . Since $\mathrm{DPP}(\mathrm{TBFu})_{2}$ serves as the donor material in the $\mathrm{BHJ} \mathrm{OPV}$, the relevant carriers are holes. Hence, the indices $i$ and $j$ represent the valence bands, and 18 highest valence bands spreading over $1.0 \mathrm{eV}$ are considered in the simulations.

The electron transition (ET) dynamics from state $i$ to $j$ can be expressed as $\operatorname{ET}(t)=1-\exp (t / \tau)$, where $\operatorname{ET}(t)=\left|c_{j}^{(\mathrm{i})}(t)\right|^{2}$ is the amount of electron transfer from state $i$ to $j$ at time $t$. $\tau$ is the characteristic time scale for the electron transfer, and $1 / \tau$ is thus the electron transition rate. The equation is valid as long as the system is in thermal equilibrium. The charge transfer determined from an NAMD trajectory of $100 \mathrm{fs}$ is fitted to the equation from which the characteristic transition time scale or rate can be estimated. Thus, 1000 such trajectories are obtained to determine the transition rates at all BOMD time steps. Since $t$ is less than 100 fs while $\tau \sim 10^{5}$ fs (cf. Figure 2), one can approximate the exponential by a linear expression $\mathrm{ET} \approx t / \tau$, and hence the transition rate $W_{i j}{ }^{0}$ can be calculated as

$$
W_{i j}^{0}=\left\langle\left. c_{j}^{(i)}(t)\right|^{2} / t\right\rangle
$$

where $\langle\ldots\rangle$ indicates an average taken over the 100 fs NAMD trajectory. 
In the entire system consisting of $100 \times 100 \times 100$ cubes, one can estimate the intercube intrinsic transition rate $W_{i j}{ }^{0}$ between state $i$ in cube $n$ and state $j^{\prime}$ in the adjacent cube $m$ from the relevant intracube transition rates as ${ }^{30,62}$

$$
W_{i j^{\prime}}{ }^{0}=\left(W_{i j}^{0}+W_{i^{\prime} j^{\prime}}{ }^{0}\right) / 2
$$

where states $i^{\prime}$ and $j$ are selected from cubes $n$ and $m$, respectively, so that the distances $R_{i j}$ and $R_{i^{\prime} j^{\prime}}$ match as closely as possible to $R_{i j^{\prime}}$. Since states $i$ and $j\left(i^{\prime}\right.$ and $\left.j^{\prime}\right)$ fall within the same cube $n(m)$, their intracube transition rates are calculated by eq 10. Finally to ensure a detailed balance for uphill-energy transitions, $\varepsilon_{i^{\prime}} \geq \varepsilon_{i}$, the thermal equilibrium transition rate $W_{i j^{\prime}}$ is defined as ${ }^{63}$

$$
W_{i j^{\prime}}=\left\{\begin{array}{c}
W_{i j^{\prime}}{ }^{0} \exp \left(-\frac{\varepsilon_{j^{\prime}}-\varepsilon_{i}-\mathrm{e} E R_{n, m}^{x}}{k_{\mathrm{B}} T}\right), \\
\text { if } \varepsilon_{j^{\prime}} \geq \varepsilon_{i}+\mathrm{e} E R_{n, m}^{x} \\
W_{i j^{\prime}}{ }^{0}, \text { if } \varepsilon_{j^{\prime}}<\varepsilon_{i}+\mathrm{e} E R_{n, m}^{x}
\end{array}\right.
$$

Here $\varepsilon_{i}$ and $\varepsilon_{j^{\prime}}$ are the energies of states $i$ and $j^{\prime}$ in cubes $n$ and $m$, respectively; $k_{\mathrm{B}}$ is the Boltzmann constant and $T$ is temperature. The term $-\mathrm{e} E R_{n, m}^{x}$ represents the energy shift in the presence of a uniform electric field $E$ in $x$ direction; $R_{n, m}^{x}$ is the distance between cube $n$ and $m$ in the $x$ direction

A similar method developed by Vukmirovic et al. ${ }^{62}$ has been used to simulate carrier mobilities in disordered semiconductors. In this approach, empirical force fields are used to determine the phonon spectrum of the material, based on which the transition rates are estimated following the perturbation theory of Fermi golden rule. In general, the validity of the empirical force fields and the perturbation theory (where weak electron-phonon coupling is assumed) has to be established before the method is applied. In contrast, our method is entirely based on first principles and thus has a broader applicability. Nonetheless, for disordered P3HT polymers, both approaches yielded a similar order of magnitude for the carrier mobility at $300 \mathrm{~K}$ [our value of $2.9 \times 10^{-5} \mathrm{~cm}^{2}$ / $(\mathrm{V} \cdot \mathrm{s})$ versus their value of $\left.0.71 \times 10^{-5} \mathrm{~cm}^{2} /(\mathrm{V} \cdot \mathrm{s})\right]$ that agreed well with the experimental result of $2.8 \times 10^{-5} \mathrm{~cm}^{2} /(\mathrm{V} \cdot \mathrm{s})$ for the regio-irregular $\mathrm{P} \mathrm{HT}^{42}$

\section{Master Equations for Mobility}

The carrier mobility is calculated for the entire system by solving the master equations for each cube $n$ in equilibrium, that is

$$
\sum_{m}\left[\Gamma_{n \rightarrow m}-\Gamma_{m \rightarrow n}\right]=0
$$

Here, $\Gamma_{n \rightarrow m}$ is the thermal equilibrium transition rate for the charge carrier from cube $n$ to cube $m$, summing up all intercube transition rates:

$$
\Gamma_{n \rightarrow m}=\sum_{i \in n} p_{n} f_{i} \sum_{j^{\prime} \in m} W_{i j^{\prime}}\left(1-p_{m} f_{j^{\prime}}\right)
$$

where $i$ and $j^{\prime}$ are summed over all relevant electronic states of the carrier in cubes $n$ and $m$, respectively; $p_{n}$ is the partial concentration or density of carriers in cube $n$, and $f_{i}$ is the Fermi-Dirac occupation of state $i$. Note that the carrier concentration $p_{n}$ is unknown and needs to be determined selfconsistently by solving the master equations. ${ }^{19}$ With the macroscopic transition rates $\Gamma_{n \rightarrow m}$, the carrier mobility $\mu$ is given by

$$
\mu=\frac{\sum_{n, m} \Gamma_{n \rightarrow m} R_{n, m}^{x}}{p E V}
$$

where $p$ is the total carrier concentration and $V$ is the volume of the entire system. In this work, we set $p=10^{-6} / \mathrm{cube}$, corresponding to a hole concentration of $1.5 \times 10^{14} \mathrm{~cm}^{-3}$, which is a typical value estimated from experiments. ${ }^{42}$

\section{AUTHOR INFORMATION}

\section{Corresponding Author}

*E-mail ganglu@csun.edu.

\section{Notes}

The authors declare no competing financial interest.

\section{ACKNOWLEDGMENTS}

The work was supported by the NSF Solar Grant (DMR1035480) and the NSF PREM Grant (DMR-1205734). We acknowledge discussions with Guillermo Bazan and Carlos Garcia-Cervera. C.F.W. is partially supported by CNPq (Brazil) fellowship (200974/2011-4).

\section{REFERENCES}

(1) Xia, P. F.; Feng, X. J.; Lu, J. P.; Tsang, S. W.; Movileanu, R.; Tao, Y.; Wong, M. S. Donor-Acceptor Oligothiophenes as Low Optical Gap Chromophores for Photovoltaic Applications. Adv. Mater. 2008, 20, 4810-4815.

(2) Silvestri, F.; Irwin, M. D.; Beverina, L.; Facchetti, A.; Pagani, G. A.; Marks, T. J. Efficient Squaraine-Based Solution Processable BulkHeterojunction Solar Cells. J. Am. Chem. Soc. 2008, 130, 1764017641.

(3) Rousseau, T.; Cravino, A.; Bura, T.; Ulrich, G.; Ziessel, R.; Roncali, J. BODIPY Derivatives as Donor Materials for Bulk Heterojunction Solar Cells. Chem. Commun. 2009, 1673-1675.

(4) Sun, Y. M.; Welch, G. C.; Leong, W. L.; Takacs, C. J.; Bazan, G. C.; Heeger, A. J. Solution-Processed Small-Molecule Solar Cells with 6.7\% Efficiency. Nat. Mater. 2012, 11, 44-48.

(5) Gunes, S.; Neugebauer, H.; Sariciftci, N. S. Conjugated PolymerBased Organic Solar Cells. Chem. Rev. 2007, 107, 1324-1338.

(6) Mandoc, M. M.; Koster, L. J. A.; Blom, P. W. M. Optimum Charge Carrier Mobility in Organic Solar Cells. Appl. Phys. Lett. 2007, 90, No. 133504.

(7) Yu, B.; Huang, L. Z.; Wang, H. B.; Yan, D. H. Efficient Organic Solar Cells Using a High-Quality Crystalline Thin Film as a Donor Layer. Adv. Mater. 2010, 22, 1017-1020.

(8) Walker, B.; Tomayo, A. B.; Dang, X. D.; Zalar, P.; Seo, J. H.; Garcia, A.; Tantiwiwat, M.; Nguyen, T. Q. Nanoscale Phase Separation and High Photovoltaic Efficiency in Solution-Processed, SmallMolecule Bulk Heterojunction Solar Cells. Adv. Funct. Mater. 2009, 19, 3063-3069.

(9) Liu, J.; Walker, B.; Tamayo, A.; Zhang, Y.; Nguyen, T. Q. Effects of Heteroatom Substitutions on the Crystal Structure, Film Formation, and Optoelectronic Properties of Diketopyrrolopyrrole-Based Materials. Adv. Funct. Mater. 2013, 23, 47-56.

(10) Street, R. A.; Northrup, J. E.; Salleo, A. Transport in Polycrystalline Polymer Thin-Film Transistors. Phys. Rev. B 2005, 71, 165202:1-13.

(11) Troisi, A. Charge Transport in High Mobility Molecular Semiconductors: Classical Models and New Theories. Chem. Soc. Rev. 2011, 40, 2347-2358.

(12) Hulea, I. N.; Fratini, S.; Xie, H.; Mulder, C. L.; Iossad, N. N.; Rastelli, G.; Ciuchi, S.; Morpurgo, A. F. Tunable Frohlich Polarons in Organic Single-Crystal Transistors. Nat. Mater. 2006, 5, 982-986. 
(13) Ostroverkhova, O.; Cooke, D. G.; Hegmann, F. A.; Anthony, J. E.; Podzorov, V.; Gershenson, M. E.; Jurchescu, O. D.; Palstra, T. T. M. Ultrafast Carrier Dynamics in Pentacene, Functionalized Pentacene, Tetracene, and Rubrene Single Crystals. Appl. Phys. Lett. 2006, 88, No. 162101.

(14) Coropceanu, V.; Cornil, J.; da Silva, D. A.; Olivier, Y.; Silbey, R.; Bredas, J. L. Charge Transport in Organic Semiconductors. Chem. Rev. 2007, 107, 926-952.

(15) Craciun, N. I.; Wildeman, J.; Blom, P. W. M. Universal Arrhenius Temperature Activated Charge Transport in Diodes from Disordered Organic Semiconductors. Phys. Rev. Lett. 2008, 100, No. 056601

(16) Troisi, A.; Orlandi, G. Charge-Transport Regime of Crystalline Organic Semiconductors: Diffusion Limited by Thermal Off-Diagonal Electronic Disorder. Phys. Rev. Lett. 2006, 96, No. 086601.

(17) Bassler, H. Charge Transport in Disordered Organic Photoconductors: A Monte-Carlo Simulation Study. Phys. Status Solidi B 1993, 175, 15-56.

(18) Hertel, D.; Bassler, H. Photoconduction in Amorphous Organic Solids. ChemPhysChem 2008, 9, 666-688.

(19) Yu, Z. G.; Smith, D. L.; Saxena, A.; Martin, R. L.; Bishop, A. R. Molecular Geometry Fluctuations and Field-Dependent Mobility in Conjugated Polymers. Phys. Rev. B 2001, 63, No. 085202.

(20) Pasveer, W. F.; Cottaar, J.; Tanase, C.; Coehoorn, R.; Bobbert, P. A.; Blom, P. W. M.; de Leeuw, D. M.; Michels, M. A. J. Unified Description of Charge-Carrier Mobilities in Disordered Semiconducting Polymers. Phys. Rev. Lett. 2005, 94, No. 206601.

(21) Bredas, J. L.; Beljonne, D.; Coropceanu, V.; Cornil, J. ChargeTransfer and Energy-Transfer Processes in Pi-Conjugated Oligomers and Polymers: A Molecular Picture. Chem. Rev. 2004, 104, 49715003.

(22) Nan, G. J.; Wang, L. J.; Yang, X. D.; Shuai, Z. G.; Zhao, Y. Charge Transfer Rates in Organic Semiconductors beyond First-Order Perturbation: From Weak to Strong Coupling Regimes. J. Chem. Phys. 2009, 130, No. 024704

(23) Koster, L. J. A. Charge Carrier Mobility in Disordered Organic Blends for Photovoltaics. Phys. Rev. B 2010, 81, No. 205318.

(24) Miller, A.; Abrahams, E. Impurity Conduction at Low Concentrations. Phys. Rev. 1960, 120, 745-755.

(25) Marcus, R. A. Electron-Transfer Reactions in Chemistry: Theory and Experiment. Rev. Mod. Phys. 1993, 65, 599-610.

(26) Vukmirovic, N.; Wang, L. W. Carrier Hopping in Disordered Semiconducting Polymers: How Accurate Is the Miller-Abrahams Model? Appl. Phys. Lett. 2010, 97, No. 043305.

(27) Munn, R. W.; Silbey, R. Theory of Electronic Transport in Molecular-Crystals.2. Zeroth Order States Incorporating Nonlocal Linear Electron-Phonon Coupling. J. Chem. Phys. 1985, 83, 18431853.

(28) Hannewald, K.; Stojanovic, V. M.; Schellekens, J. M. T.; Bobbert, P. A.; Kresse, G.; Hafner, J. Theory of Polaron Bandwidth Narrowing in Organic Molecular Crystals. Phys. Rev. B 2004, 69, No. 075211.

(29) Kenkre, V. M.; Andersen, J. D.; Dunlap, D. H.; Duke, C. B. Unified Theory of the Mobilities of Photoinjected Electrons in Naphthalene. Phys. Rev. Lett. 1989, 62, 1165-1168.

(30) Zhang, X.; Li, Z.; Lu, G. First-Principles Determination of Charge Carrier Mobility in Disordered Semiconducting Polymers. Phys. Rev. B 2010, 82, No. 205210.

(31) Li, Z.; Zhang, X.; Lu, G. A Fortran Program for Calculating Electron or Hole Mobility in Disordered Semiconductors from FirstPrinciples. Comput. Phys. Commun. 2011, 182, 2632-2637.

(32) Duncan, W. R; Stier, W. M.; Prezhdo, O. V. Ab Initio Nonadiabatic Molecular Dynamics of the Ultrafast Electron Injection Across the Alizarin- $\mathrm{TiO}_{2}$ Interface. J. Am. Chem. Soc. 2005, 127, 79417951.

(33) Li, Z.; Zhang, X.; Lu, G.; Nguyen, T. Q. First-Principles Study of Electron Mobility in Cationic and Anionic Conjugated Polyelectrolytes. J. Phys. Chem. C 2012, 116, 1205-1210.
(34) Curtis, M. D.; Cao, J.; Kampf, J. W. Solid-State Packing of Conjugated Oligomers: From Pi-Stacks to the Herringbone Structure. J. Am. Chem. Soc. 2004, 126, 4318-4328.

(35) Kobayashi, K.; Shimaoka, R.; Kawahata, M.; Yamanaka, M.; Yamaguchi, K. Synthesis and Cofacial Pi-Stacked Packing Arrangement of 6,13-Bis(alkylthio)pentacene. Org. Lett. 2006, 8, 2385-2388.

(36) Wang, C. L.; Dong, H. L.; Li, H. X.; Zhao, H. P.; Meng, Q.; Hu, W. P. Dibenzothiophene Derivatives: From Herringbone to Lamellar Packing Motif. Cryst. Growth Des. 2010, 10, 4155-4160.

(37) Lampert, M.; Mark, P., Current Injection in Solids; Academic: New York, 1970.

(38) van Mensfoort, S. L. M.; Coehoorn, R. Effect of Gaussian Disorder on the Voltage Dependence of the Current Density in Sandwich-Type Devices Based on Organic Semiconductors. Phys. Rev. B 2008, 78, No. 085207.

(39) Koster, L. J. A.; Smits, E. C. P.; Mihailetchi, V. D.; Blom, P. W. M. Device Model for the Operation of Polymer/Fullerene Bulk Heterojunction Solar Cells. Phys. Rev. B 2005, 72, No. 085205.

(40) Woellner, C. F.; Freire, J. A. Reformulated Space-ChargeLimited Current Model and Its Application to Disordered Organic Systems. J. Chem. Phys. 2011, 134, No. 084112.

(41) Wetzelaer, G. A. H.; Koster, L. J. A.; Blom, P. W. M. Validity of the Einstein Relation in Disordered Organic Semiconductors. Phys. Rev. Lett. 2011, 107, No. 066605.

(42) Tanase, C.; Meijer, E. J.; Blom, P. W. M.; de Leeuw, D. M. Unification of the Hole Transport in Polymeric Field-Effect Transistors and Light-Emitting Diodes. Phys. Rev. Lett. 2003, 91, No. 216601.

(43) Abkowitz, M.; Bassler, H.; Stolka, M. Common Features in the Transport Behavior of Diverse Glassy Solids: Exploring the Role of Disorder. Philos. Mag. B 1991, 63, 201-220.

(44) Blom, P. W. M.; deJong, M. J. M.; vanMunster, M. G. ElectricField and Temperature Dependence of the Hole Mobility in Poly ( $p$ phenylene vinylene). Phys. Rev. B 1997, 55, R656-R659.

(45) Campbell, I. H.; Smith, D. L.; Neef, C. J.; Ferraris, J. P. Consistent Time-of-Flight Mobility Measurements and Polymer LightEmitting Diode Current-Voltage Characteristics. Appl. Phys. Lett. 1999, 74, 2809-2811.

(46) Liu, J.; Mikhaylov, I. A.; Zou, J.; Osaka, I.; Masunov, A. E.; McCullough, R. D.; Zhai, L. Insight into How Molecular Structures of Thiophene-Based Conjugated Polymers Affect Crystallization Behaviors. Polymer 2011, 52, 2302-2309.

(47) Ko, S.; Hoke, E. T.; Pandey, L.; Hong, S.; Mondal, R.; Risko, C.; Yi, Y.; Noriega, R.; McGehee, M. D.; Bredas, J. L.; Salleo, A.; Bao, Z. Controlled Conjugated Backbone Twisting for an Increased OpenCircuit Voltage while Having a High Short-Circuit Current in Poly(hexylthiophene) Derivatives. J. Am. Chem. Soc. 2012, 134, 5222-5232.

(48) Craciun, N. I.; Brondijk, J. J.; Blom, P. W. M. DiffusionEnhanced Hole Transport in Thin Polymer Light-Emitting Diodes. Phys. Rev. B 2008, 77, No. 035206.

(49) Tanase, C.; Blom, P. W. M.; de Leeuw, D. M. Origin of the Enhanced Space-Charge-Limited Current in Poly( $p$-phenylene vinylene). Phys. Rev. B 2004, 70, No. 193202.

(50) Dang, X. D.; Tamayo, A. B.; Seo, J.; Hoven, C. V.; Walker, B.; Nguyen, T. Q. Nanostructure and Optoelectronic Characterization of Small Molecule Bulk Heterojunction Solar Cells by Photoconductive Atomic Force Microscopy. Adv. Funct. Mater. 2010, 20, 3314-3321.

(51) Kim, C.; Liu, J. H.; Lin, J. S.; Tamayo, A. B.; Walker, B.; Wu, G.; Nguyen, T. Q. Influence of Structural Variation on the Solid-State Properties of Diketopyrrolopyrrole-Based Oligophenylenethiophenes: Single-Crystal Structures, Thermal Properties, Optical Bandgaps, Energy Levels, Film Morphology, and Hole Mobility. Chem. Mater. 2012, 24, 1699-1709.

(52) Bozano, L.; Carter, S. A.; Scott, J. C.; Malliaras, G. G.; Brock, P. J. Temperature- and Field-Dependent Electron and Hole Mobilities in Polymer Light-Emitting Diodes. Appl. Phys. Lett. 1999, 74, 11321134. 
(53) Nicolai, H. T.; Mandoc, M. M.; Blom, P. W. M. Electron Traps in Semiconducting Polymers: Exponential versus Gaussian Trap Distribution. Phys. Rev. B 2011, 83, No. 195204.

(54) Mandoc, M. M.; de Boer, B.; Paasch, G.; Blom, P. W. M. TrapLimited Electron Transport in Disordered Semiconducting Polymers. Phys. Rev. B 2007, 75, No. 193202.

(55) Kuik, M.; Wetzelaer, G.-J. A. H.; Ladde, J. G.; Nicolai, H. T.; Wildeman, J.; Sweelssen, J.; Blom, P. W. M. The Effect of Ketone Defects on the Charge Transport and Charge Recombination in Polyfluorenes. Adv. Funct. Mater. 2011, 21, 4502-4509.

(56) Hwang, W.; Kao, K. C. Studies of the Theory of Single and Double Injections in Solids with a Gaussian Trap Distribution. SolidState Electron. 1976, 19, 1045-1047.

(57) Lampert, M. A. Simplified Theory of Space-Charge-Limited Currents in an Insulator with Traps. Phys. Rev. 1956, 103, 1648-1656. (58) Nicolai, H. T.; Kuik, M.; Wetzelaer, G. A. H.; de Boer, B.; Campbell, C.; Risko, C.; Bredas, J. L.; Blom, P. W. M. Unification of Trap-Limited Electron Transport in Semiconducting Polymers. Nat. Mater. 2012, 11, 882-887.

(59) Perdew, J. P.; Burke, K.; Ernzerhof, M. Generalized Gradient Approximation Made Simple. Phys. Rev. Lett. 1996, 77, 3865-3868.

(60) Kresse, G.; Hafner, J. Ab Initio Molecular Dynamics for Liquid Metals. Phys. Rev. B 1993, 47, 558-561.

(61) Kresse, G.; Furthmuller, J. Efficient Iterative Schemes for Ab Initio Total-Energy Calculations Using a Plane-Wave Basis Set. Phys. Rev. B 1996, 54, 11169-11186.

(62) Vukmirovic, N.; Wang, L. W. Charge Carrier Motion in Disordered Conjugated Polymers: A Multiscale Ab Initio Study. Nano Lett. 2009, 9, 3996-4000.

(63) Duncan, W. R; Craig, C. F.; Prezhdo, O. V. Time-Domain Ab Initio Study of Charge Relaxation and Recombination in DyeSensitized $\mathrm{TiO}_{2}$. J. Am. Chem. Soc. 2007, 129, 8528-8543. 